\title{
Image Segmentation Algorithm Based on the AIC
}

\author{
G.Y. Chen \\ Department of Mathematics \\ Dalian Maritime University \\ Dalian, Liaoning, China \\ N.N. Liu \\ Department of Mathematics, \\ Dalian Maritime University, \\ Dalian, Liaoning, China
}

\author{
H.Y. Xie \\ Department of Mathematics \\ Dalian Maritime University \\ Dalian, Liaoning, China
}

\author{
D.Q. Liang \\ School of Information Science and Technology \\ Dalian Maritime University \\ Dalian, Liaoning, China
}

\begin{abstract}
Clustering-based segmentation algorithm is one of the common methods in image segmentation. However, how to avoid the influence of the initialization and the singular value is a problem we have to face. For overcoming this problem, we proposed a new image segmentation technology which is based on the AIC criterion and not affected by the singular values. The simulation results show that the method has good performance on stability and accuracy.
\end{abstract} AIC

Keywords- segmentation; cluster analysis; K-L information;

\section{INTRODUCTION}

Image segmentation is the key step in image analysis of computer vision and image processing field. It has been widely applied in various areas such as in the process of automating production, sensing images and biomedical images. The main goal of image segmentation is to simplify an image into segments that have a strong correlation with objects in the real world. The existing segmentation algorithm can be generally divided into three classes: edge detection, regionbased segmentation and data clustering. Edge detection method relies on the edges of the object with background and the other objects. For the images with noise, it is difficult to separate the boundary and the target background [1]. Regionbased segmentation method segments an image into several meaningful sub-domains that are non-overlapping and samenature. But it may lead to the over-segmentation, and has to combine with other methods to use [2]. Data clustering method is based on the whole image and considers the distance between each data. Advantages of data clustering method are low complexity and easy to implement. Disadvantages are sensitive to the noise and selection of the initial centroids [3]. Data clustering is one of the common techniques in image segmentation. Its purpose is to cluster pixels into several parts (regions) according to the feature of image. Based on the properties of clustering algorithm, researchers have proposed various image segmentation algorithm, such as based on hierarchical clustering, based on $K$-means clustering, based on fuzzy clustering, based on mean shift, and so on [3-5].

Clustering analysis is an unsupervised process of partitioning a data set into subsets of similar data objects. The elementary principle is as far as possible to increase the "difference" between categories and reduce the "difference" within the category. In the process of image segmentation based on data clustering, thus the objective is that the similar pixels are divided into the same regions and the non-similar pixels are divided into different regions. However, Two difficulties we must face are that how to determine the cluster centers and avoid influence of noise.

In this paper, a new image segmentation algorithm is proposed based on the AIC criterion. The new segmentation technology can achieve an ideal performance and not affected by the initialization of centroids and the singular values. The simulation results verify the efficiency of proposed algorithm.

\section{AIC CRITERION}

The Akaike Information Criterion (AIC Criterion), developed by Hirotsugu Akaike [6], is a way of selecting a model from a set of models. The chosen model is the one that minimizes the Kullback-Leibler distance between the model and the truth.

Supposing $X$ is a random variable with the distribution density function $g(x)$ belongs to the family of distribution function $\{f(x \mid \theta), \theta \in \Theta\}$. Let $\theta^{*}$ be the true value of $\theta$ and $g(x)=f\left(x \mid \theta^{*}\right)$. According to the principle of maximum likelihood, $L(\theta ; x)$ achieves the maximum at the point $\theta=\theta^{*}$ which means that $\log \{g(x) / f(x \mid \theta)\}$ achieves the minimum at the point $\theta=\theta^{*}$. According to the ensemble average to consider extremum, the Kulblack Lehbler information ( $\mathrm{K}$ - L information) is defined as follows.

Definition 1. Let

$$
I[g(\cdot) ; f(\cdot \mid \theta)]=E[\log (g(x))]-E[\log (f(x \mid \theta))]=-E\left\{\log \left(\frac{f(x \mid \theta)}{g(x)}\right)\right\}
$$

Then $I[g(\bullet) ; f(\bullet \mid \theta)]$ is called the K-L information.

About K-L information, we have the following property. 
Lemma

$$
I[g(\cdot) ; f(\cdot \mid \theta)] \geq \mathbf{0}
$$

$I[g(\cdot) ; f(\cdot \mid \theta)]=\mathbf{0}$ if $\theta=\theta^{*}$.

Proof :

$$
\begin{aligned}
& I[g(\cdot) ; f(\cdot \mid \theta)]=-E\{\log [f(x \mid \theta) / g(x)]\} \\
& =-\log \int[f(x \mid \theta) / g(x)] \cdot g(x) d x \\
& =-\log \int f(x \mid \theta) d x \\
& =-\log 1 \\
& =0
\end{aligned}
$$

The proof is completed.

Obviously, K-L information can characterize the degree of the similarity between $f(x \mid \theta)$ and $\boldsymbol{g}(\boldsymbol{x})$. The larger the value of K-L information implies the bigger the difference between $f(x \mid \theta)$ and $g(x)$. So, K-L information can find the parameter density function $f(x \mid \theta)$ of the closest $g(x)$. When $\theta=\theta^{*}$, the value of K-L information is 0 which is the best degree of fitting between $f(x \mid \theta)$ and $g(x)$. AIC criterion is based on this principle.

\section{Definition 2 .}

ACI $=-2$ (The maximum likelihood function of the model) +2 (The number of the free parameters in the model)

The criterion of selecting the model is to minimize the AIC value. According to the definition of the AIC, there are two differences in the model. The first case is that the difference between the models is mainly on the second item of the equation if the model has the same maximum likelihood. When using AIC to choose the models, the model with less free parameters is better. That is the "principle of saving". The second case is that the difference between the models is mainly on the first item of the equation if the model has the same parameter. It tends to choose the model with large maximum likelihood. Based on the above two cases, AIC has the minimum value.

\section{APPLICATION OF AIC CRITERION IN THE IMAGE SEGMENTATION}

AIC criterion has been used in many fields since the AIC criterion was proposed by Akaike in 1973. In this paper, the AIC criterion will be applied to in the area of image segmentation[7].

Supposing $R$ represents an image with the size $M \times N$ and we denote $n=M \times N$. The image segmentation is dividing $n$ sample points into $m$ groups by taking each pixel point of the image recorded as a sample point. Suppose that each group of the data has the independent and identically distributtions $f\left(\cdot \mid \theta_{i}\right)$ and denote the $\mathrm{j}$ sample of the $\mathrm{k}$ group recorded as $x_{k j}$. Let $g(\cdot)_{i}=f\left(\cdot \mid \theta_{k}^{*}\right), k=1,2, \ldots m$, and $n_{1}+n_{2}+\ldots n_{m}=n$, where $\theta_{k}^{*}$ is the true value of probability density parameters of the $\mathrm{k}$ group. In this article, we take the gray image as an example and suppose that the data of $\mathrm{k}$ group come from sample of overall the normal population $N\left(\mu_{k}, \sigma_{k}^{2}\right)$. Then the number of free parameters is 2 for each group. we calculate the $\mu_{k}, \sigma_{k}^{2}$ as follows:

$$
\begin{aligned}
& \hat{\mu}_{k}=\frac{1}{n_{i}} \sum_{j-1}^{n_{i}} x_{i j}, \hat{\sigma}_{k}^{2}=\frac{1}{n_{i}} \sum_{j-1}^{n_{i}}\left(x_{i j}-\hat{\mu}_{i}\right)^{2} . \\
& \text { Where } \quad L\left(\hat{\mu}, \sigma^{2}\right)=\sum_{i-1}^{m} \sum_{j-1}^{n_{i}} \log f\left(x_{i j} \mid \hat{\mu}, \hat{\sigma}^{2}\right) \quad \text { is }
\end{aligned}
$$

Likelihood function of each group of the data. By applying AIC criterion, we have

$$
\operatorname{AIC}(m)=-2 L\left(\hat{\mu}, \hat{\sigma}^{2}\right)+2 K=\sum_{i=1}^{m} n_{i} \log \hat{\sigma}_{i}^{2}+4 m+n(1+\log (2 \pi))
$$

Since $n(1+\log (2 \pi))$ is constant, we can simplify as $A I C(m)=\sum_{i=1}^{m} n_{i} \log \hat{\sigma}_{i}^{2}+4 m$.

Then, the image segmentation is finding a classification result minimize the value of $A I C(m)$ for the given of clustering number. The explanation of this method is that the first item of AIC reflect the difference of the two classification results. As the first item is the sum of the logarithm of variance of data, the $A I C(m)$ acchieived the minimum when the classification results the difference within class reaching the minimum. Then the AIC has the optimal value which is corresponding to the $\mathrm{s}$ most suitable classification.

\section{THE EXPERIMENTAL RESULTS}

Taking Figure 1 as the original image and setting the clustering number as 3, 4, 5 resepctively. Table 1 shows the division node and Figure 2-4 shows the Segmentation results.

TABLE I. SHOWS THE DIVISION NODE.

\begin{tabular}{|l|l|l|l|l|l|}
\hline $\mathbf{m}$ & $\boldsymbol{A I C}(\boldsymbol{m})$ & Note 1 & Note 2 & Note 3 & Note 4 \\
\hline 3 & 1136.6 & 63 & 126 & & \\
\hline $\mathbf{4}$ & 1031.4 & 47 & 95 & 142 & \\
\hline $\mathbf{5}$ & 950.8578 & 38 & 76 & 114 & 152 \\
\hline
\end{tabular}

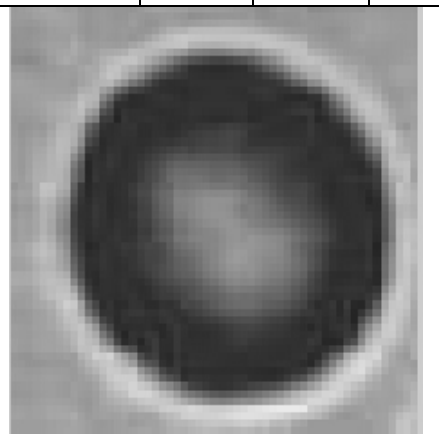

FIGURE I. THE ORIGINAL IMAGE. 


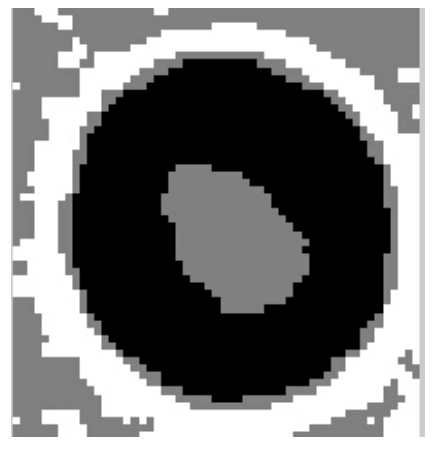

FIGURE II. THE NUMBER OF GROUPS OF CLASSIFICATION IS 3.

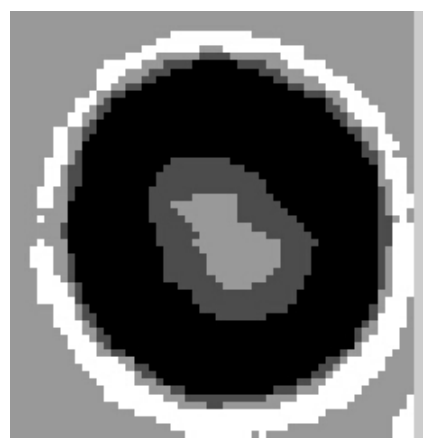

FIGURE III. THE NUMBER OF GROUPS OF CLASSIFICATION IS 4.

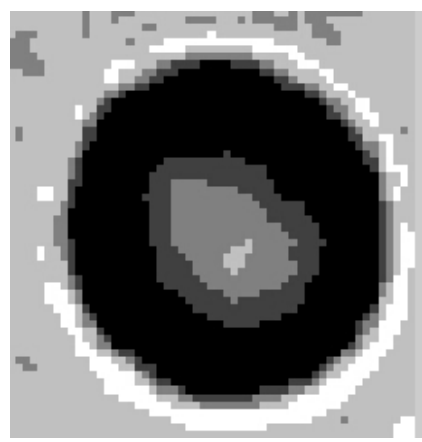

FIGURE IV.THE NUMBER OF GROUPS OF CLASSIFICATION IS 5

\section{CONCLUSIONS}

AIC criterion is applied to images segmentation in this article. The each pixel of the image is taking as a random variable and each set of data after the image segmentation is taking as normal distribution. The algorithm can effectively avoid the influence of singular value on the result of image segmentation. The minimum value of ACI is the criterion of model selection. In this paper, we assume that the cluster number of segmentation is given, so then the differences of image segmentation mainly come from the first item of the AIC criterion.

Indeed, according to the definition of the AIC, the difference between the models is mainly reflected in the second of the equation when the maximum likelihood is same. Select the model with the AIC as a standard would tend to choose the model of free parameters less. From the point of image segmentation, we can make the further discussion by using AIC to adaptive segmentation to select the appropriate classification number.

\section{ACKNOWLEDGMENT}

This work is partially supported by the Fundamental Research Funds for the Central Universities 3132013094.

\section{REFERENCES}

[1] A.X.Falcao, J.K.Udupa, F.K.Miyazawa, An Ultra-Fast User-Steered Image Segmentation Paradigm: Live Wire on the Fly [J]. IEEE Transactions on Medical Imaging, 2000, 19(1): 55-62.

[2] Matalas L, Benjamin R, Kitney R, An Edge Detection Technique Using the Facet Model and Parameterized Relaxation Labeling [J]. IEEE Transactions Trans on Pattern Analysis and Machine Intelligence, 1997, 19(4): 328-341.

[3] Yu-Hsiang Wang. Tutorial: Image Segmentation, http://disp.ee.ntu.edu.tw/ meeting/\%E6\%98\%B1\%E7\%BF\%94/Segmentation\%20tutorial.pdf, Dec 19, 2010.

[4] Keh-Shih Chuang, Hong-Long Tzeng, Sharon Chen, Jay Wu, Tzong-Jer Chen. Fuzzy C-Means Clustering with Spatial Information for Image Segmentation [J]. Computerized Medical Imaging and Graphics, 2006, 30(1): 9-15.

[5] Z.H.Cui, S.Sun, S.G.Chen, L.Q.Gao. Mean Shift Based FCM Image Segmentation Algorithm [J], Control and Decision, 2014, 29(6): 11301134.

[6] Akaike. H. A New Look at the Stochastic Model Identification. IEEE Transactions on Automatic Control [J], 2003, 19(6): 716-723.

[7] X.Y.Qin. Nearest Neighbor Clustering Algorithm Based on AIC Criterion. Systems Engineering and Electronics, 2005, 27(2): 257-259. 